\title{
Danish national clinical guidelines for surgical and nonsurgical treatment of patients with lumbar spinal stenosis
}

\author{
Rikke Rousing ${ }^{1} \cdot$ Rikke Krüger Jensen ${ }^{4,5,7} \cdot$ Søren Fruensgaard ${ }^{2}$. Janni Strøm ${ }^{2,3} \cdot$ Helle Algren Brøgger ${ }^{5,7,8}$.

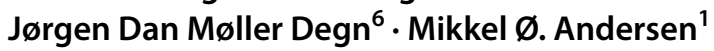

Received: 21 December 2017 / Revised: 6 March 2019 / Accepted: 19 April 2019 / Published online: 16 May 2019

(c) The Author(s) 2019

\begin{abstract}
Purpose To summarize the recommendations from the national clinical guideline published by the Danish Health Authority regarding surgical and nonsurgical interventions in treatment of lumbar spinal stenosis in patients above the age of 65 years. Methods A multidisciplinary working group formulated recommendations based on the GRADE approach.

Results Seven of the recommendations were based on randomized controlled trials and three on professional consensus. The guideline recommends surgical decompression for symptomatic lumbar spinal stenosis. Supervised exercise and postsurgical supervised exercise are recommended due to the general beneficial effects of training on general health, even though there was no evidence on an effect on neurogenic pain. The guideline does not recommend manual therapy, paracetamol, NSAIDs, opioids, neurogenic pain medication, muscle relaxants, and decompression combined with instrumented fusion as there was no evidence of the beneficial effect.

Conclusion The recommendations are based on low to very low quality of evidence or professional consensus as well as patient preferences and positive or harmful effects of the intervention. The true treatment effect may therefore be different from the estimated effects, which is why the results should be interpreted with caution. The working group recommends intensified research in relation to all aspects of management of lumbar spinal stenosis.
\end{abstract}

\section{Graphical abstract}

These slides can be retrieved under Electronic Supplementary Material.
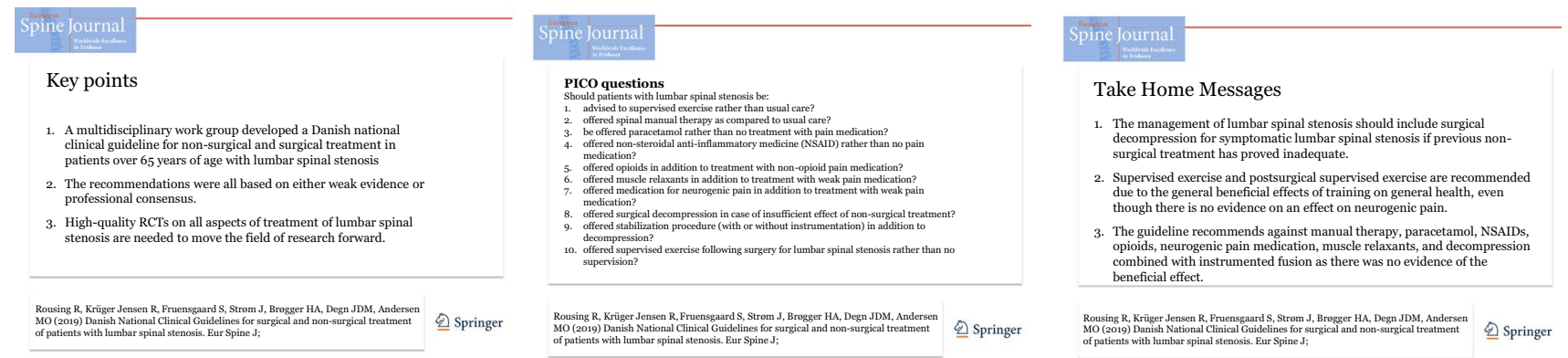

Keywords Lumbar spinal stenosis $\cdot$ Guideline $\cdot$ Surgical and nonsurgical interventions

Electronic supplementary material The online version of this article (https://doi.org/10.1007/s00586-019-05987-2) contains supplementary material, which is available to authorized users.

Rikke Rousing

rikke.rousing@rsyd.dk

Extended author information available on the last page of the article

\section{Introduction/background}

In 2012, the Danish Finance Act (DFA) devoted 11 million Euros to the development of national clinical guidelines. The Danish Health Authority (DHA) was requested to lead the work of publishing national clinical guidelines on different health areas to support evidence-based decision making 
and uniform high-quality treatment across Denmark. One of these health areas was lumbar spinal stenosis (LSS) which was chosen due to the large variation in treatment practice in the different regions of Denmark and because of the high burden of disease.

Although the prevalence of LSS is uncertain, it is considered a very common condition among elderly patients. In a study by Ishimoto et al. [1], the authors reported the prevalence of symptomatic LSS to be $10 \%$ in a Japanese general population (mean age 66) and increasing with age. In Denmark, nearly $30 \%$ of the population will be over the age of 60 in the year 2030 [2] and the burden of disease is therefore expected to further increase.

LSS can cause significant pain and disability leading to dramatically reduced quality of life, immobility, and functional limitation. Also, a recent study by Ammendolia et al. [3] investigated the physical and psychological impact of LSS from patients' perspectives and found that in addition to pain and limited walking ability, the emotional effects of the disability was impactful to the patients' lives.

Degenerative LSS is caused by age-related changes in the spine, such as arthrosis of the facet joints, thickening of the ligamentum flavum, and bulging of dehydrated disks. Thereby, the spinal canal is narrowed which may result in symptoms due to neural compression such as radiating pain, numbness and weakness, diminished walking capacity, and balance disturbances. The symptoms are often worsened by standing and walking and relieved by sitting or lying down. When crouching while walking, the symptoms may be diminished [4]. In addition, patients with LSS very often have concurrent back pain although this is not specific for LSS. The diagnosis is based on the presence of these symptoms often obtained from the case history and maybe combined with primarily MRI or with CT if MRI is contraindicated.

Furthermore, standing anterior-posterior and lateral radiographs are sometimes used as a supplement to uncover spinal instability and presurgical planning in patients in whom bony anatomy needs to be illustrated accurately [5].

The treatment of patients suffering from LSS is diverse. Some patients are offered pain medications, some are offered nonsurgical treatment such as exercise and manual treatment, and some are recommended decompression surgery of the neural structures with or without concomitant fusion of the spinal segment.

The purpose of this paper was to summarize the Danish national clinical guideline published in Danish September 2017 [6] for treatment modalities of LSS in patients above 65 years of age. The mandate of the working group was to make recommendations on a maximum of ten interventions covering different treatment options both nonsurgical and surgical, to ensure uniform high-quality treatment of patients with LSS.

\section{Materials and methods}

\section{Study design}

The clinical guideline was based on a critical systematic review of the scientific literature followed by meta-analyses. The final recommendations were a balance between the evidence available and potential risk of harms together with patient preference. A detailed DHA handbook [7] of the method was followed based on the Grades of Recommendation, Assessment, Development, and Evaluation (GRADE) approach. The full clinical guideline in Danish is available on the webpage of DHA [6].

\section{Description of the work process}

A project group of employees from DHA consisting of a chairman, a project manager, a lead reviewer, a search specialist, a methodologist, and a multidisciplinary work group was formed. The work group members were appointed by invitation from professional organizations and scientific societies and were composed of two neurosurgeons, two orthopedic surgeons, one rheumatologist, one anesthesiologist, one geriatrician, one radiologist, one nurse, two physiotherapists, one chiropractor, and one medical practitioner. Potential conflict of interest was published on the DHA homepage [8]. The work group members participated in formulating the ten research questions for evaluation, grading the literature, and formulating the recommendations. A reference group with representatives from the Danish public healthcare system and patient organizations approved the clinical questions and gave advice on the recommendations.

The lead reviewer coordinated the work and drafted the report.

\section{Treatment questions}

The work group agreed on ten research questions concerning nonsurgical and surgical treatment of LSS to be considered.

The research questions were structured according to the PICO framework in which the questions are divided into four components (population, intervention, comparison, and outcome) [9].

\section{Population}

This study was restricted to patients with LSS verified with relevant radiological examination. Additionally, the target group was characterized by significant symptoms such as radiating pain, numbness, balance disturbances, diminished 
walking capacity, limited function and loss of activity of daily living, and worsening of the symptoms by standing and walking and relieved in rest.

Patients with other kinds of radiating leg pain such as vascular claudication, hip arthrosis, and disk herniation were excluded. Patient populations with radiological signs of spinal instability were excluded.

Even though LSS can be present in all age groups (although prevalence increases with age), the guideline was targeted on patients above the age of 65 years.

\section{Intervention}

The intervention included nonsurgical intervention, medical intervention, and surgical intervention.

\section{Comparison}

Nonsurgical intervention was compared to either no treatment or to usual care, which was not specified any further to avoid exclusion of relevant literature. Medical intervention was compared to no treatment or to treatment with nonopioid pain medication. Surgical treatment was compared to no treatment or nonsurgical treatment.

\section{Outcome}

For each research question, outcomes measures were determined. Up to four of these were determined to be the primary outcome measures. For most questions leg pain, walking distance, and physical disability were the primary outcomes. In the surgical research questions, surgical complications were included as a primary outcome. Other outcome measures comprised of quality of life, risk of falling, the need for pain medication, and side effects to medication. Outcomes were divided into short-term follow-up defined as $0-12$ weeks and long-term follow-up defined as 6-18 months.

\section{Literature review}

Search parameters and terms were determined by the multidisciplinary group, and a literature search was completed by a specialist from DHA according to the search protocols. The following electronic databases were searched for English, Scandinavian, and German language publications from 2006 to 2016: Medline, Embase, PsycInfo, CINAHL, and Pedro. The search terms and strategies are available at the DHA website [10].

The literature search was performed in three steps: The first search was performed on clinical guidelines, the second on systematic reviews, and the third on randomized controlled trials. The lead reviewer screened and retrieved the titles and abstracts. Potentially eligible papers were collected in full text and a work group member and the lead reviewer independently screened the papers for inclusion and exclusion criteria. In case of disagreements, the paper was discussed to achieve consensus.

The best research evidence available was used to answer the PICO questions. Clinical guidelines were evaluated according to AGREE, systematic reviews according to AMSTAR, and randomized studies according to Cochrane Risk of Bias Tool.

In case of high-quality clinical guidelines or systematic reviews, data were extracted directly from these studies. Data from clinical randomized studies were extracted by the lead reviewer and a work group member independently. Disagreements on the data extraction were solved by consensus. The handling of references and data extractions was performed by the use of the web-based software Covidence. Data were exported to the RevMan software, and meta-analyses were performed by the methodologist.

The quality of the evidence was graded according to the GRADE definition: very low, low, moderate, or high (Table 1).

\section{Creation of recommendations}

The evidence was presented to the work group in tables, and forest plots were presented when meta-analyses were performed. From the available evidence, strong or weak recommendation either for or against a treatment was suggested (Table 2).

Table 1 Definitions of grades of recommendation, assessment, development, and evaluation (GRADE) adapted from Balshem et al. 2011

\begin{tabular}{ll}
\hline Quality of evidence & Definition \\
\hline $\begin{array}{l}\text { High }(\oplus \oplus \oplus \oplus) \\
\text { Moderate }(\oplus \oplus \oplus \mathrm{O})\end{array}$ & $\begin{array}{l}\text { We are very confident that the true effect lies close to the estimate of the effect } \\
\text { We are moderately confident of the estimated effect: The true effect is likely to be close to the estimate, but there is a } \\
\text { possibity that is substantially different } \\
\text { We have limited confidence of estimated effect: The true effect may be substantially different from the estimated effect } \\
\text { Very low }(\oplus \mathrm{OOO})\end{array}$ \\
$\begin{array}{l}\text { We have very little confidence in the estimated effect: The true effect is likely to be substantially different from the } \\
\text { estimate }\end{array}$
\end{tabular}


Table 2 Recommendations and their definitions by the Danish Health Authority (DHA)

\begin{tabular}{|c|c|}
\hline Recommendation & Definition \\
\hline Strong recommendation in favor $\uparrow \uparrow$ & $\begin{array}{l}\text { The DHA makes a strong recommendation in favor of an intervention when its desirable effect } \\
\text { clearly outweighs undesirable effect }\end{array}$ \\
\hline Weak/conditional recommendation in favor $\uparrow$ & $\begin{array}{l}\text { The DHA makes a weak/conditional recommendation in favor of an intervention when the } \\
\text { desirable effect of an intervention is considered to outweigh the undesirable effects or when } \\
\text { the available evidence cannot rule out a significant benefit of an intervention and the side } \\
\text { effects are judged to be few or absent }\end{array}$ \\
\hline Weak/conditional recommendation against $\downarrow$ & $\begin{array}{l}\text { The DHA makes a weak/conditional recommendation against an intervention when the undesir- } \\
\text { able effects are judged to outweigh the desirable effects, but where this is not supported by } \\
\text { strong evidence } \\
\text { This recommendation is also made in case of strong evidence for both beneficial and harm- } \\
\text { ful effects when the equilibrium between them is difficult to determine. Also used when it is } \\
\text { likely that patient preferences vary }\end{array}$ \\
\hline Strong recommendation against $\downarrow \downarrow$ & $\begin{array}{l}\text { The DHA makes a strong recommendation against an intervention in case of high-quality } \\
\text { evidence showing that the undesirable effects of an intervention clearly outweigh the desirable } \\
\text { effects, or when the review of the evidence demonstrates great certainty that the intervention } \\
\text { is ineffective }\end{array}$ \\
\hline Good practice $\sqrt{ }$ & $\begin{array}{l}\text { Good practices are based on professional consensus among the members of the working group. } \\
\text { The recommendation may be either for or against the intervention. These types of recommen- } \\
\text { dations are weaker than the evidence-based recommendations irrespective of whether these } \\
\text { are strong or weak }\end{array}$ \\
\hline
\end{tabular}

In lack of evidence, expert opinion reached by discussion in the work group was the basis for practical recommendation.

The final recommendations were based on the evidence available together with potential positive or negative effects as well as patient preference. When the recommendations were established, work group members developed the guideline content, referencing the literature that supported the recommendations.

\section{Results}

In total, ten clinical questions were covered. Nonsurgical treatment for LSS was covered by two research questions concerning supervised exercise and manual therapy, while one other question addressed rehabilitation after surgery for LSS. Pharmacological treatment was covered in five questions concerning the use of paracetamol, NSAIDs, opioids, muscle relaxants, and neurogenic pain medication. Two questions covered the surgical treatment concerning decompression and fusion. A summary of the interventions and recommendations is presented in Table 3 .

None of the PICO questions could be answered by means of existing clinical guidelines or reviews (step one and two in the literature search). The recommendations were therefore based on RCTs for seven of the PICO questions and the remaining three on professional consensus. All RCTs were graded as low or very low quality mostly due to indirectness, small study sample, and imprecision.
Only one RCT was identified comparing supervised exercise as treatment for LSS to patient education and information [11]. The study found no statistical significant differences between the two interventions for any of the outcomes. Due to the general benefits of exercise and patient preference, the final recommendation was in favor of supervised exercise.

One RCT compared manual therapy and muscle strength exercise to flexion exercise and walking training and found no statistical significant differences on outcomes except for the treatment-satisfaction subscale of Zurich Claudication Questionnaire improvement in favor of manual therapy, although the effect size did not reach the predefined criterion for clinical relevance [12]. As there are no known general benefits of manual therapy, the final recommendation was not in favor of manual therapy based on the lack of knowledge in the literature.

Postsurgical supervised exercise was compared to home exercise or no exercise in two RCTs [13, 14]. No differences were found in any of the outcomes, but due to the general benefits of exercise and patient preference, the recommendation favored postsurgical supervised exercise.

Treatment with pain medication for LSS was covered by five PICO questions in which the specific pain medication was compared to no pain medication or to pain medication with non-opioid medication.

No studies concerning nonsteroidal anti-inflammatory drugs (NSAIDs), paracetamol, or muscle relaxants were identified. The final recommendations based on consensus did not favor any of the three pain medications primarily due to the lack of evidence and risk of side effects. 
Table 3 PICO questions, recommendations, definitions of interventions, supporting evidence, and comments regarding lumbar spinal stenosis

PICO 1. Should patients with lumbar spinal stenosis be advised to supervised exercise rather than usual care?

$\uparrow$ Consider offering supervised training to patients with spinal stenosis due to the general beneficial effects of training on health and the lack of known adverse effects. $(\oplus \mathrm{OOO})$
Definition: Supervised exercise was defined as exercises or physical activities, either as general health and fitness or as specific back-exercise, e.g., strengthening, mobilizing, stretching, balance, motor control and cardiovascular exercise. The program must take place under the guidance of a relevant healthcare professional, and be targeted and adapted specifically to the individual level

Included studies: For supervised exercise, we identified one RCT [11] comparing supervised exercise with information and education

Primary outcomes: The following outcomes were investigated: SSS, Physical Function, ODI and the number of shuttles the patients can complete (walking test). There were no significant differences in the effect of the intervention between the two groups and no clinically relevant improvement in the two groups

Comment: The quality of the evidence was overall very low. The level of evidence was downgraded as there was only one small study. Due to the general benefits of exercise, the final recommendation favored exercise, although focus was suggested to be on cardiovascular exercise as there was no evidence found in favor of exercise as treatment for neurogenic pain

PICO 2. Should patients with lumbar spinal stenosis be offered spinal manual therapy as compared to usual care?

$\downarrow$ Spinal manual therapy should not be used routinely for patients with lumbar spinal stenosis, since the beneficial effect is uncertain. $(\oplus \mathrm{OOO})$

Definition: Spinal manual therapy was defined as any manual technique that moves one or more lumbar joint segments or pelvic joints including mobilization and high velocity spinal manipulative techniques

Included studies: We identified one RCT [12] in which spinal manual therapy (thrust and non-thrust manipulation, manual spine stretching and muscle strengthening exercises) was compared to flexion and walking exercises

Primary outcomes: The following outcomes were investigated: ODI, NPRS ZCQ satisfaction, and Walking distance. There was a minimal statistically significant improvement in the manipulation group compared to the control group in ZCQ satisfaction sub-score $(0.46$ after 6 weeks and 0.26 after 1 year). However, the effect size was not considered clinically relevant according to the predefined cutpoints. There were no statistically significant differences in the other outcomes

Comment: The quality of the evidence was overall very low. The level of evidence was downgraded due to only one small study and indirectness

PICO 3. Should patients with lumbar spinal stenosis be offered paracetamol rather than no treatment with pain medication?

$\sqrt{ }$ It is good practice to avoid use of paracetamol in patients with lumbar spinal stenosis, since the beneficial effect is uncertain and undocumented
Definition: Pain medication in the form of paracetamol (max 4 grams daily) with treatment duration from 4 to 12 weeks

Included studies: No studies

Comment: The potential adverse effects of paracetamol, e.g., liver damage in case of unknown renal disorder versus the lack of evidence of beneficial effects on neurogenic pain has resulted in a good practice recommendation against the use of paracetamol in patients with symptomatic lumbar spinal stenosis

PICO 4. Should patients with lumbar spinal stenosis be offered nonsteroidal anti-inflammatory medicine (NSAID) rather than no pain medication?

$\sqrt{ }$ It is good practice to avoid use of NSAIDs in patients with lumbar spinal stenosis since the beneficial effect is uncertain and there is a risk of adverse reactions
Definition: Anti-inflammatory drugs in the form of NSAIDs, such as ibuprofen 400-600 mg 3-4 times or naproxen $250-500 \mathrm{mg}$ twice daily with treatment duration from 4 to 12 weeks.

Included studies: No studies

Comments: The potential adverse effects of NSAID treatment, e.g., gastrointestinal bleeding and cardiac side effects versus the lack of evidence of beneficial effects on neurogenic pain led to a recommendation against the use of NSAIDs in patients with symptomatic lumbar spinal stenosis 
Table 3 (continued)

PICO 5. Should patients with lumbar spinal stenosis be offered opioids in addition to treatment with non-opioid pain medication?

$\downarrow$ Opioids should only be used for patients with lumbar spinal stenosis upon due consideration, since the beneficial effect is uncertain and there is a risk of adverse reactions. $(\oplus \mathrm{OOO})$
Definition: Opioids, e.g., morphine $10 \mathrm{mg}$ 3-4 times daily, oxycodone $5-10 \mathrm{mg}$ twice a day or tramadol 50-100 mg 3-4 times daily in addition to non-opioid pain medication and with a treatment duration from 4 to 12 weeks

Included studies: We identified one study [17] in which patients were randomized into one of three groups: (i) oxymorphone hydrochloride $5 \mathrm{mg}$, (ii) placebo or (iii) propoxyphene/acetaminophen. The patients received a single dose of the given substance in a random order separated by a 3 -day leaching period. The study was discontinued before its completion because of serious side effects to propoxyphene/ acetaminophen

Primary outcomes: The primary outcome was the time until the patients' experienced moderate pain in a walking test. Other outcomes were ZCQ, symptom severity and physical function RMDQ, ODI, walking disturbance and cerebral side effects. There was no significant difference between the three treatments

Comments: The quality of the evidence is overall very low. The level of evidence was downgraded based on the fact that there is only one small study. In addition the intervention differs from the above definition. The frequent and bothering side effects associated with opioid treatment and the lack of evidence of beneficial effect on neurogenic pain is the basis for the weak recommendation against treatment with opioids in patients with symptomatic lumbar spinal stenosis

PICO 6. Should patients with lumbar spinal stenosis be offered muscle relaxants in addition to treatment with weak pain medication?

$\sqrt{ }$ It is good practice to avoid use of muscle relaxants in patients with lumbar spinal stenosis, since the beneficial effect is uncertain and there is a risk of adverse reactions
Definition: Muscle relaxants, e.g., tizanidin 2-4 mg 3-4 times a day or chlorzoxazone $250 \mathrm{mg} \mathrm{3-4} \mathrm{times} \mathrm{a} \mathrm{day} \mathrm{in} \mathrm{addition} \mathrm{to} \mathrm{treatment} \mathrm{with}$ weak pain relievers. Duration of treatment 4-12 weeks. Benzodiazepines are not considered relevant in this context Included studies:

Comments: The potential adverse side effects of muscle relaxants, e.g., dizziness, fatigue, dry mouth, muscle weakness and gastrointestinal side effects, as well as the lack of evidence of beneficial effects on neurogenic pain is the basis for a good practice recommendation against the use of muscle relaxants in patients with symptomatic lumbar spinal stenosis

PICO 7. Should patients with lumbar spinal stenosis be offered medication for neurogenic pain in addition to treatment with weak pain medication?

$\downarrow$ Medical treatment of neurogenic pain should only be initiated in patients with lumbar spinal stenosis upon due consideration, since the beneficial effect is uncertain and there is a risk of adverse reactions $(\oplus \mathrm{OOO})$
Definition: Medication for neurogenic pain, e.g., Gabapentin $300 \mathrm{mg}$ three times a day, increasing to $900 \mathrm{mg}, 3$ times a day

Included studies: We identified two studies regarding neurogenic pain medication. One study [16] compared gabapentin to placebo and the other study [15] compared pregabalin to placebo

Primary outcomes: In the study by Yaksi, the pain level and walking distance were measured. In the study by Markmann, RMDQ, ODI, SSS were additionally measured. Gabapentin did relieve pain and increases walking distance statistically significant compared to placebo, but this was not consistent across follow-up. There was not difference between groups for pregabalin

Comments: The quality of the evidence is overall very low. The level of evidence was downgraded as there were only two small studies, the type of medicine was different in the two studies, and there were indirectness
With regard to medication for neurogenic pain, two randomized studies were identified $[15,16]$. One study comparing gabapentin to placebo found a statistical significant improvement in leg pain and walking distance in favor of gabapentin, but this was not a consistent finding on all time points, and the effect size did not reach clinical significance. 
Table 3 (continued)

PICO 8. Should patients with lumbar spinal stenosis be offered surgical decompression in case of insufficient effect of nonsurgical treatment?

$\uparrow$ Consider surgical decompression surgery to patients with lumbar spinal stenosis if previous nonsurgical treatment has proved inadequate. $(\oplus \oplus \mathrm{OO})$
Definition: Surgical decompression, partial hemi-laminectomy or broad decompression

Included studies: We identified three RCTs concerning surgical decompression [18-20] compared to either exercise or patient information and education

Primary outcomes:

The studies addressed ODI, walking distance and leg pain. The studies did not report on any side effects. The studies are characterized by cross-over, especially from the nonsurgical arm to the decompression arm. In the study of Weinstein, both intention-to-treat and as-treated analyzes have been performed, and in the latter analysis, the results of a nonrandomized cohort are also included

The intention-to-treat analysis did not show statistical significant results on any of the outcomes. However, the as-treated analysis found a statistical significant association in favor of decompression on both pain and ODI, although, the ODI did not reach the predefined cut-point for a clinical relevant difference

Comments:

The quality of the evidence is overall low. The level of evidence was downgraded because of the included cohort study of Weinstein and due to inconsistent results

PICO 9. Should patients with lumbar spinal stenosis be offered stabilization procedure (with or without instrumentation) in addition to decompression?

$\downarrow$ Fusion surgery should only be used as an add-on to decompression for patients with lumbar spinal stenosis upon due consideration, since the beneficial effect is uncertain. $(\oplus \oplus \mathrm{OO})$

Definition: Stabilization (with or without instrumentation) in addition to surgical decompression

Included studies: We identified one study comparing laminectomy with instrumented stabilization procedure to laminectomy only [21]. Included patients had been diagnosed with stable spondylolistesis. Another study by Forsth et al. was identified; however, this was not included as the follow-up was more than one year which was the predefined limit in this guideline. No studies on un-instrumented fusion surgery were identified

Primary outcomes:

The study addressed ODI, quality of life (SF-36), and side effects. There was a statistical significant improvement in SF-36 in favor of instrumented fusion, but not in ODI. The study reported fewer reoperations in the instrumented fusion group. There are no statistical significant differences in the complication rates between the two groups

Comments: The quality of the evidence is overall low. The level of evidence was downgraded because of only one study and low mean age

PICO 10. Should patients be offered supervised exercise following surgery for lumbar spinal stenosis rather than no supervision?

$\uparrow$ Consider offering supervised rehabilitation to patients who have undergone surgery for lumbar spinal stenosis, due to the general beneficial effects of training and the lack of known adverse effects. $(\oplus \oplus \mathrm{OO})$
Definition: Supervised exercise was defined as exercises or physical activities, either as general health and fitness or as specific back-exercise, e.g., strengthening, mobilizing, stretching, balance, motor control and cardiovascular exercise. The program must take place under the guidance of a relevant healthcare professional, and be targeted and adapted specifically to the individual level

Included studies: We identified two RCTs comparing supervise exercise to either home exercise or no exercise $[13,14]$. Two other studies were identified but not included as for one study [28] it was not possible to extract data and for the other [29] exercise was initiated presurgical

Primary outcomes: The studies addressed ODI, pain, and walking distance and quality of life. No statistical significant difference was found on any of the outcome measures

Comments: The quality of the evidence is overall low. The level of evidence is downgraded because of only one small study and high variability. In the study by McGregor et al. patients with lateral nerve compression are included which introduces indirectness

SSS Swiss spinal stenosis questionnaire, ZCQ Zurich claudication questionnaire, SF-36 Short Form 36, NPRS The Numeric Pain Rating Scale, $R M D Q$ Roland Morris Disability Questionnaire, $O D I$ Oswestry Disability Index 
The other study showed no difference between pregabalin and placebo in leg pain, walking distance, and clinical outcomes. Because of this lack of evidence and a significant risk of side effects, the recommendation did not favor neurogenic pain medication.

One RCT was identified in which two different opioids (oxymorphone hydrochloride and propoxyphene/acetominophen) were compared to placebo [17]. The study was terminated early due to serious side effects to propoxyphene/ acetominophen. The study showed no statistical significant differences between the three medications in pain and physical function. Because of lack of evidence and the risk of side effects, the recommendation did not favor opioids.

Surgical treatment for LSS was covered by two PICOs: decompression and supplementing decompression with fusion either instrumented or noninstrumented.

Surgical decompression was addressed in three RCTs [18-20]. In all studies, decompression was compared to exercise or general advice. All studies showed a large proportion of cross-over particular from the nonsurgical arm to the surgical arm, (43\% after 2 years in the study by Weinstein and $57 \%$ after 2 years in the study by Delitto). In the study by Weinstein, both intention-to-treat analysis and as-treated analysis were performed, and in the as-treated analysis, the results from a cohort study were included. Overall, the studies showed statistical significant improvement in leg pain and ODI in favor of decompression, but predefined cut-points for clinical significance were not reached. The largest effect was seen when including the as-treated analysis, and the smallest effect was seen in the intention-to-treat analysis. The final recommendation was in favor of surgical decompression due to the statistically significant and partly clinical relevant improvement in leg pain and ODI.

Surgical decompression supplemented with instrumented stabilization was identified in one study by Ghogawala et al. [21]. No studies on noninstrumented stabilization were identified. In the study by Ghogwala et al., patients were randomized to either decompression or decompression supplemented with instrumented stabilization. All patients in this study had been diagnosed with stable spondylolisthesis. The study reported a statistical significant improvement in quality of life measured with SF-36, but not in the ODI. The number of patients needing reoperation was lower in the treatment arm with additional stabilization. The final recommendation did not favor instrumented stabilization on patients with stable spondylolisthesis due to the lack of relevant clinical difference in outcome compared to decompression without stabilization.

\section{Discussion}

Patients with significant symptoms due to LSS such as radiating pain, numbness, balance disturbances, diminished walking capacity, low back pain, limited function, and loss of activity of daily living verified with relevant radiological examination might benefit from supervised exercise. As we found, no randomized studies showing significant better outcome in patients receiving supervised exercise compared to usual care; the treatment should focus on general conditioning. Manual therapy may be considered for patients with spinal stenosis in case of concomitant low back pain, but has no clinical effect on neurogenic pain. In addition, manual therapy has no effect on general conditioning.

NSAIDs and paracetamol were the only drugs investigated which may be considered for a short time in case of concomitant low back pain. We found no evidence for the effect of the pharmacological treatment options investigated in this guideline on neurogenic pain. The national clinical guideline 'Diagnosis and Treatment of Degenerative Lumbar Spinal Stenosis' from North American Spine Society (2011) [22] also found insufficient evidence to make a recommendation for or against the use of pharmacological treatment for patients with LSS. Guidelines on sciatica (radicular neurogenic pain) from both the National Institute of Health Care Excellence (NICE) in the UK (2016) [23] and Danish National Guidelines (DNG) (2016) [24] recommend against routine use of paracetamol and that NSAIDs is used in the lowest effective dose and only after considering the risk for side effects for the individual patient. Also, they recommend that opioids should not be used routinely. Patients with LSS are older than patients with sciatica considered in the guidelines from NICE and DNG and therefore in higher risk of comorbidity and side effects which should be carefully considered when recommending pharmacological treatment.

Surgical decompression should be considered as the treatment option for symptomatic LSS in case of serious symptoms for longer than 3-6 months. Care should be taken to inform the patients about general possible complications associated with decompression, increased risk due to possible comorbidity, and expected outcome [25]. In case of spinal instability, instrumented or noninstrumented spinal fusion could be considered.

Despite the great number of patients with LSS, there is a lack of RCTs concerning this patient population. There are no or only few studies investigating the effect of medical treatment for LSS, and only few studies explore the effect of nonsurgical treatment. The number of studies concerning surgery for spinal stenosis is slightly bigger, but in these studies, the cross-over from one arm to 
the other, and especially from the nonsurgical arm to the surgical arm, is found in more than $50 \%$, which tends to diminish the treatment effect of decompression. In the study by Weinstein et al. [20], the as-treated analysis was performed to avoid the cross-over problem, but thereby, the effect of randomization is no longer present. In addition to that, the results were combined with the results from a cohort study, and this is blurring the effect of the randomization even more.

Nevertheless, the RCTs included in this guideline concerning decompression surgery show the largest improvement for patients with symptomatic LSS compared to other treatment modalities. Stabilization procedure does not seem to increase the effect based on very-low quality evidence. However, only patients with stable spondylolisthesis were included in the study by Ghogawala et al., and the results might have been different had the patient population included instable spondylolisthesis. The recommendation against spinal fusion is supported by a highquality RCT by Försth with two and five years follow-up (not included in this guideline) [26].

The major strength of this guideline was the adherence to the relevant scientific standards, and the work was assisted by expert research librarians and guideline methodologists. Also, the working group was composed of clinicians with a wide range of professional background ensuring a broad forum for discussion which was important due to the lack of evidence. The weakness of this work was the lack of clinical trials in most areas, with the consequence that several of the recommendations were reached by consensus in the working group. Also, the recommendations based on the literature were based on either few or only one clinical trial. Overall, the workgroup found a substantial lack of evidence for the effectiveness of the interventions addressed. Therefore, new high-quality trials are likely to impact the recommendations in future guidelines. However, the GRADE working group encourages to make recommendations even though they are not based on solid evidence [27]. In addition, the guideline only addresses 10 PICO questions and had a national focus which means that other treatment options such as epidural steroid injections were not covered in this guideline.

It is recommended that the guideline is updated three years after publication and that guideline recommendations are implemented in national and regional patient course descriptions for primary and secondary care. Collection of process and effect indicators could be achieved by creating a nationwide, multidisciplinary spinal registry for the primary and secondary care units. To move the field of research forward, high-quality RCTs on all aspects of treatment of LSS are needed including research in defining phenotypes of LSS.

\section{Conclusion}

A multidisciplinary work group developed a national clinical guideline for nonsurgical and surgical treatment in patients over 65 years of age with LSS. The recommendations were all based on either weak evidence or professional consensus.

The guideline work group recommends that the management of LSS should include surgical decompression for symptomatic LSS. Supervised exercise and postsurgical supervised exercise are recommended due to the general beneficial effects of training on general health, even though there is no evidence on an effect on neurogenic pain. The guideline recommends against manual therapy, paracetamol, NSAIDs, opioids, neurogenic pain medication, muscle relaxants, and decompression combined with instrumented fusion as there was no evidence of the beneficial effect.

Summary of recommendations

Guideline recommendations by the Danish Health Authority:

1. The management of lumbar spinal stenosis should include surgical decompression for symptomatic lumbar spinal stenosis

2. Decompression combined with instrumented fusion is not indicated as there is no evidence of beneficial effect in the stable spine

3. All actions known to be beneficial on general health like supervised exercise and postsurgical supervised exercise are recommended even though there is no evidence on an effect on neurogenic pain

4. The guideline recommends against manual therapy, paracetamol, NSAIDs, opioids, neurogenic pain medication, muscle relaxants

Acknowledgements We would like to acknowledge the working group: Ane Just Ohrt (chairman), Assistant Medical Officer, the Danish Health Authority, Anne Keller, senior consultant, Rigshospitalet-Glostrup, Allan Horn, senior consultant, Zealand University Hospital Køge, Frauke Wolfram, senior consultant, Rigshospitalet-Glostrup, Gert Vedel Sørensen, senior consultant, Odense University Hospital, Helle Algren Brøgger, physiotherapist, Lillebaelt Hospital, Janni Strøm, nurse, Regionshospitalet Silkeborg, Jørgen Dan Møller Degn, senior consultant, Rigshospitalet-Glostrup, Mikkel Østerheden Andersen, senior consultant, Lillebaelt Hospital, Niels Bro Madsen, medical practitioner, Solrød Strand, Ole Fedders, senior consultant, Aalborg University Hospital, Rikke Krüger Jensen, chiropractor, Lillebaelt Hospital, Simon Simonsen, physiotherapist, Vejle Rygklinik, Søren Fruensgaard, senior consultant, Regionshospitalet Silkeborg. We would also like to thank Kirsten Birkefoss, research librarian, the Danish Health Authority, Jens Aabo, Method Consultant, the Danish Health Authority and Maria Herlev Ahrenfeldt (project manager), Specialist Adviser, the Danish Health Authority, for their contributions.

\section{Compliance with ethical standards}

Conflict of interest Potential conflicts of interest have been declared by all involved partners and made publicly available on the Danish Health Authority webpage (in Danish). 
Open Access This article is distributed under the terms of the Creative Commons Attribution 4.0 International License (http://creativeco mmons.org/licenses/by/4.0/), which permits unrestricted use, distribution, and reproduction in any medium, provided you give appropriate credit to the original author(s) and the source, provide a link to the Creative Commons license, and indicate if changes were made.

\section{References}

1. Ishimoto $\mathrm{Y}$, Yoshimura N, Muraki S, Yamada H, Nagata K, Hashizume $\mathrm{H}$ et al (2012) Prevalence of symptomatic lumbar spinal stenosis and its association with physical performance in a population-based cohort in Japan: the Wakayama Spine Study. Osteoarthr Cartil 20(10):1103-1108

2. www.dst.dk/enj. Statistics Denmark

3. Ammendolia C, Schneider M, Williams K, Zickmund S, Hamm M, Stuber K et al (2017) The physical and psychological impact of neurogenic claudication: the patients' perspectives. J Can Chiropr Assoc 61(1): 18-31

4. Tomkins-Lane C, Melloh M, Lurie J, Smuck M, Battie MC, Freeman B et al (2016) ISSLS prize winner: consensus on the clinical diagnosis of lumbar spinal stenosis: results of an international Delphi Study. Spine 41(15):1239-1246

5. Segebarth B, Kurd MF, Haug PH, Davis R (2015) Routine upright imaging for evaluating degenerative lumbar stenosis: incidence of degenerative spondylolisthesis missed on supine MRI. J Spinal Disord Tech 28(10):394-397

6. https://www.sst.dk/da/nyheder/2017/ /media/24A94AF7B421465 8AF47DD8A87A420B3.ashx. National clinical guideline for the treatment of lumbar spinal stenosis

7. www.sst.dk/da/nkr/metode/ /media/A11D669470D04B70B776 16DBA92CF985.ashx. Metodehåndbog for udarbejdelse af Nationale Kliniske Retningslinjer for puljeprojekter 2017-2020

8. www.sst.dk/da/om-os/maal-og-opgaver/habilitet/soeg-habilitets erklaering? search $=\{802030$ F9-1134-4570-9013-6FFDB 18900 A1\}. habilitetserklæring

9. Guyatt GH, Oxman AD, Kunz R, Atkins D, Brozek J, Vist G et al (2011) GRADE guidelines: 2. Framing the question and deciding on important outcomes. J Clin Epidemiol 64(4):395-400

10. www.sst.dk/da/udgivelser/2017/ /media/BDF82BCD24EA4B0 AA8D5E645C857B52A.ashx. Search terms and strategies

11. Comer C, Redmond AC, Bird HA, Hensor EM, Conaghan PG (2013) A home exercise programme is no more beneficial than advice and education for people with neurogenic claudication: results from a randomised controlled trial. PLoS ONE 8(9):e72878

12. Whitman JM, Flynn TW, Childs JD, Wainner RS, Gill HE, Ryder MG et al (2006) A comparison between two physical therapy treatment programs for patients with lumbar spinal stenosis: a randomized clinical trial. Spine 31(22):2541-2549

13. McGregor AH, Dore CJ, Morris TP, Morris S, Jamrozik K (2011) ISSLS prize winner: function after spinal treatment, exercise, and rehabilitation (FASTER): a factorial randomized trial to determine whether the functional outcome of spinal surgery can be improved. Spine 36(21):1711-1720

14. Aalto TJ, Leinonen V, Herno A, Alen M, Kroger H, Turunen V et al (2011) Postoperative rehabilitation does not improve functional outcome in lumbar spinal stenosis: a prospective study with 2-year postoperative follow-up. Eur Spine J 20(8):1331-1340

15. Markman JD, Frazer ME, Rast SA, McDermott MP, Gewandter JS, Chowdhry AK et al (2015) Double-blind, randomized, controlled, crossover trial of pregabalin for neurogenic claudication. Neurology 84(3):265-272

16. Yaksi A, Ozgonenel L, Ozgonenel B (2007) The efficiency of gabapentin therapy in patients with lumbar spinal stenosis. Spine 32(9):939-942

17. Markman JD, Gewandter JS, Frazer ME, Murray NM, Rast SA, McDermott MP et al (2015) A randomized, double-blind, placebo-controlled crossover trial of oxymorphone hydrochloride and propoxyphene/acetaminophen combination for the treatment of neurogenic claudication associated with lumbar spinal stenosis. Spine 40(10):684-691

18. Delitto A, Piva SR, Moore CG, Fritz JM, Wisniewski SR, Josbeno DA et al (2015) Surgery versus nonsurgical treatment of lumbar spinal stenosis: a randomized trial. Ann Intern Med 162(7):465-473

19. Slatis P, Malmivaara A, Heliovaara M, Sainio P, Herno A, Kankare J et al (2011) Long-term results of surgery for lumbar spinal stenosis: a randomised controlled trial. Eur Spine J 20(7):1174-1181

20. Weinstein JN, Tosteson TD, Lurie JD, Tosteson AN, Blood E, Hanscom B et al (2008) Surgical versus nonsurgical therapy for lumbar spinal stenosis. N Engl J Med 358(8):794-810

21. Ghogawala Z, Dziura J, Butler WE, Dai F, Terrin N, Magge SN et al (2016) Laminectomy plus fusion versus laminectomy alone for lumbar spondylolisthesis. N Engl J Med 374(15):1424-1434

22. Kreiner DS, Shaffer WO, Baisden JL, Gilbert TJ, Summers JT, Toton JF et al (2013) An evidence-based clinical guideline for the diagnosis and treatment of degenerative lumbar spinal stenosis (update). Spine J 13(7):734-743

23. Bernstein IA, Malik Q, Carville S, Ward S (2017) Low back pain and sciatica: summary of NICE guidance. BMJ 356:i6748

24. Stochkendahl MJ, Kjaer P, Hartvigsen J, Kongsted A, Aaboe J, Andersen $\mathrm{M}$ et al (2018) National Clinical Guidelines for nonsurgical treatment of patients with recent onset low back pain or lumbar radiculopathy. Eur Spine J 27(1):60-75

25. Zweig T, Enke J, Mannion AF, Sobottke R, Melloh M, Freeman $\mathrm{BJC}$ et al (2017) Is the duration of pre-operative conservative treatment associated with the clinical outcome following surgical decompression for lumbar spinal stenosis? A study based on the Spine Tango Registry. Eur Spine J 26(2):488-500

26. Forsth P, Olafsson G, Carlsson T, Frost A, Borgstrom F, Fritzell $P$ et al (2016) A randomized, controlled trial of fusion surgery for lumbar spinal stenosis. N Engl J Med 374(15):1413-1423

27. Andrews J, Guyatt G, Oxman AD, Alderson P, Dahm P, FalckYtter Y et al (2013) GRADE guidelines: 14. Going from evidence to recommendations: the significance and presentation of recommendations. J Clin Epidemiol 66(7):719-725

28. Mannion AF, Denzler R, Dvorak J, Muntener M, Grob D (2007) A randomised controlled trial of post-operative rehabilitation after surgical decompression of the lumbar spine. Eur Spine $\mathbf{J}$ 16(8):1101-1117

29. Chen CY, Chang CW, Lee ST, Chen YC, Tang SF, Cheng CH et al (2015) Is rehabilitation intervention during hospitalization enough for functional improvements in patients undergoing lumbar decompression surgery? A prospective randomized controlled study. Clin Neurol Neurosurg 129(Suppl 1):S41-S46

Publisher's Note Springer Nature remains neutral with regard to jurisdictional claims in published maps and institutional affiliations. 


\section{Affiliations}

\section{Rikke Rousing ${ }^{1} \cdot$ Rikke Krüger Jensen $^{4,5,7} \cdot$ Søren Fruensgaard ${ }^{2} \cdot$ Janni Strøm $^{2,3} \cdot$ Helle Algren Brøgger ${ }^{5,7,8}$.

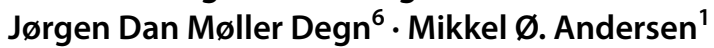

1 Center for Spine Surgery and Research, Lillebaelt Hospital, Oestre Hougvej 55, 5500 Middelfart, Denmark

2 Department of Orthopaedic Surgery, Regionshospitalet Silkeborg, Hospitalsenhed Midt, Falkevej 1-3, 8600 Silkeborg, Denmark

3 Department of Public Health, Section for Clinical Social Medicine and Rehabilitation, Aarhus Universtity, Bartholins Allé 2, 8000 Arhus, Denmark

4 Nordic Institute of Chiropractic and Clinical Biomechanics, University of Southern Denmark, Campusvej 55, 5230 Odense M, Denmark
5 Research Department, Spine Centre of Southern Denmark, Lillebaelt Hospital, Oestre Hougvej 55, 5500 Middelfart, Denmark

6 Center for Rheumatology and Spine Diseases, Rigshospitalet-Glostrup, Nordre Ringvej 57, 2600 Glostrup, Denmark

7 Department of Regional Health Research, Faculty of Health Sciences, University of Southern Denmark, Winsløwparken 13, 3, 5000 Odense C, Denmark

8 University College South Denmark, Degnevej 16, 6705 Esbjerg $\varnothing$, Denmark 